\title{
Glycocalyx Gone Awry: Pathologic Cell Signaling during Endotheliopathy
}

\author{
Robert P Richter ${ }^{1}$ and Jillian R Richter ${ }^{2 *}$ \\ ${ }^{1}$ Department of Pediatrics; University of Alabama at Birmingham, USA \\ ${ }^{2}$ Department of Surgery; University of Alabama at Birmingham, USA
}

*Corresponding author: Jillian R Richter; University of Alabama at Birmingham; Alabama, USA.

To Cite This Article: Jillian Richter. Glycocalyx Gone Awry: Pathologic Cell Signaling during Endotheliopathy. Am J Biomed Sci \& Res. 2019 - 5(2). AJBSR.MS.ID.000891. DOI: 10.34297/AJBSR.2019.05.000891.

Received: 眥 September 03, 2019; Published: 此 September 16, 2019

\begin{abstract}
The endothelial glycocalyx is a viscous mesh that lines the vascular lumen and serves a variety of functions in health and disease. This gelatinous matrix of proteoglycans and glycosaminoglycans is the critical contact point between the circulation and end organs, transmitting physical and biochemical signals through the endothelial surface layer to affect endothelial cell activity that subsequently impacts organ function. In this review we explore the roles of the major structural components of the glycocalyx in mediating cell signaling events during inflammatory-induced endotheliopathy. We also discuss proposed therapeutic strategies to attenuate glycocalyx disruption and promote its regeneration.

Keywords: Vascular Endothelium; Heparan Sulfate; Hyaluronan; Syndecan; Glypican; Inflammation; Heparanase; Matrix Metalloproteinase

Abbreviations: Agpt-2: Angiopoietin-2; ATIII: Antithrombin III; CD44: Cluster of Differentiation 44; CS: Chondroitin Sulfate; eNOS: Endothelial Nitric Oxide Synthase; ESL: Endothelial Surface Layer GAG: Glycosaminoglycan; GCX: Glycocalyx; Erk: Extracellular Signal-Regulated Kinase; FGF: Fibroblast Growth Factor; FGFR: Fibroblast Growth Factor Receptor; HA: Hyaluronan; HMW: High Molecular Weight; HPSE: Heparanase; HS: Heparan Sulfate; HSPG: Heparan Sulfate Proteoglycan; ICAM-1: Intercellular Adhesion Molecule-1; IL-1 $\beta$ : Interleukin-1 Beta; LMW: Low Molecular Weight; MAP kinase: Mitogen-Activated Protein Kinase; MMP: Matrix Metalloproteinase; NF-kB: Nuclear Factor Kappa-Light-Chain Enhancer Of Activated B Cells; NO: Nitric Oxide; PECAM-1: Platelet Endothelial Cell Adhesion Molecule-1; PI3K: Phosphoinositide-3-Kinase; PKCo: Protein Kinase C Alpha; PKC $\delta$ : Protein Kinase C Delta; RHAMM: Receptor For Hyaluronan Mediated Motility; ROS: Reactive Oxygen Species; Syn: Syndecan; TLR: Toll-Like Receptor; VCAM-1: Vascular Cell Adhesion Molecule-1; VE-cadherin: VEGF: Vascular Endothelial Growth Factor; VEGFR: Vascular Endothelial Growth Factor Receptor
\end{abstract}

\section{Introduction}

The vascular endothelial glycocalyx (GCX) is the interface of blood-endothelial interactions and an active regulator of vascular tone and permeability. Overwhelming inflammatory states like trauma, sepsis, and burns precipitate a cascade of events that culminate in multiorgan dysfunction, primarily through endothelial cell activation and GCX disruption. Degradation of the GCX has been shown to increase vascular permeability, resulting in tissue edema; propagate local inflammation; and activate microvascular coagulation with resultant microthrombi and vascular occlusion. Though much attention has been dedicated to understanding the pathophysiologic mechanisms leading to GCX disintegrity, our understanding of the precise endothelial cell signaling mechanisms affected by GCX degradation and their implications on vascular homeostasis remains incomplete. Our focus in this review will be on the known and posited downstream extra- and intracellular signaling events that contribute to vascular dysfunction after endothelial GCX disruption secondary to inflammation. We will also discuss the work that has been done to therapeutically target these mechanisms in an effort to restore vascular integrity.

\section{Background}

The GCX of the vascular endothelium is a matrix of luminal cellsurface proteoglycans (e.g., syndecan, glypican, versican, decorin, mimecan, biglycan) decorated with a variety of glycosaminoglycans (GAG) (e.g., heparan sulfate, hyaluronic acid, chondroitin sulfate, dermatan sulfate, and keratan sulfate) [1,2]. The thickness and composition of the endothelial GCX vary significantly across tissues, tailoring the function of the endothelial surface layer (ESL) to its particular native tissue and local blood flow [1,3-5].

The major structural proteoglycans in the vascular ESL are the membrane-anchored heparan sulfate proteoglycans (HSPG) syndecan and glypican[1]. Syndecans (Syn) are a transmembrane family of four proteoglycans (Syn1-Syn4) that uniquely cooperate with a host of surface growth receptors and transmit extracellular 
signaling to the intracellular environment through their highly conserved transmembrane domain[6]. Nuanced intracellular communications are dictated by the conserved C1 and C2 and variable $\mathrm{V}$ cytoplasmic domains of the Syn protein that associate with distinct enzymes and cytoskeletal elements [6]. The extracellular ectodomain of Syn is much more variable in its length compared to the cytoplasmic domain and predominantly expresses heparan sulfate, though post-translational substitutions with chondroitin sulfate moieties may also be expressed [6]. The diverse expression of HS lengths and sulfation patterns on Syn allows the GCX to serve as a large reservoir of associated plasma proteins and participate in a wide array of cell signaling pathways [7]. Glypicans only express an extracellular domain that is anchored to the endothelial cell membrane through a glycosylphosphatidylinositol anchor imbedded in lipid rafts and caveolae [8,9]. The remaining proteoglycans are expressed in the ESL as secreted molecules within the GCX and contribute significantly less its structure and function [10].

The most ubiquitously expressed GAGs in the endothelial GCX are heparan sulfate and hyaluronan [11]. Heparan sulfate (HS) is a highly variable GAG covalently bound to Syn and glypican on the endothelial cell surface and comprises over $50 \%$ of the GAG composition in the GCX. [11] The diversity of glucosamine and hexuronic acid (i.e., iduronic acid or glucuronic acid) polymerization and varied sulfation pattern allows the HS molecule to associate with a host of plasma proteins with specificity [12]. Imbedded within the HSPG mesh of the GCX are cytokines and chemokines that form gradients for endothelial cell signaling and leukocyte chemotaxis [7]. Additionally, the interaction of growth factors with HS on parent HSPGs governs the activation of the parent cell surface growth factor receptors[6]. Hyaluronan, or hyaluronic acid (HA), exists as a high molecular weight (HMW) GAG (over 1MDa) attached to the ESL through interactions with CD44 and other GAGs[11]. Its function is related to its uniquely non-sulfated, very large structure that provides both a lubricating and structurestabilizing role in the GCX. As a relative proportion, the expression of chondroitin, dermatan, and keratan sulfates is approximately four times less than that of heparan sulfate [11]. Dermatan sulfate (also classified as chondroitin sulfate type B) and keratan sulfate are primarily bound to secreted proteoglycans and exist more prevalently in the extracellular matrix of endothelial cells [11]. These lesser understood GAGs appear to contribute little to ESL function.

In health, the intact GCX serves several vital homeostatic functions. The negatively charged, highly proteinaceous composition of the GCX provides an electrostatic and physical barrier to circulating proteins to limit their extravasation and concomitantly limit the flow of circulating water to the extravascular space via modified Starling forces [13]. Endothelial cell surface receptors are buried within this luminal matrix and rely on proteoglycans and GAGs as co-receptors for cell signal activation [14]. Circulating cell interactions with the endothelium are regulated by cytokine and chemokine gradients within the GCX and by the various cell adhesion molecules hidden within the GCX $[15,16]$. The ESL also contains expressed cofactors for coagulation homeostasis, including antithrombin III (ATIII), thrombomodulin, tissue factor, and tissue factor pathway inhibitor. Thus, the GCX serves as a key touchpoint between and regulator of systemic inflammation and coagulation. Moreover, the gelatinous-like nature of the GCX transmits shear stress and hydrostatic pressure to the endothelial cells, resulting in endothelial nitric oxide synthase (eNOS) and nitric oxide (NO) regulation that coordinates vascular tone and permeability [17].

In diseases of local and systemic inflammation, degradation of the GCXresults in a predictable cascade ofinflammation propagation, coagulation activation, and increased vascular permeability. Due to the intricate involvement of the endothelial GCX in the cell surface signaling, disruption of the GCX results in significant perturbations in extra- and intracellular signaling pathways that propagate the pathophysiology of the inflamed state. The components of the GCX contribute in unique ways. Elucidating the individual contributions of the GCX components to the pathophysiology of endotheliopathy could prove instrumental in the development of successful therapies that restore vascular integrity in the setting of systemic inflammation.

\section{Role of Heparan Sulfate}

In adults with respiratory failure or sepsis, circulating HS levels are significantly elevated compared to controls and correlate with illness severity $[18,19]$. Plasma HS levels are also higher in patients after traumatic injury [20] or major vascular procedures that induce systemic ischemia/reperfusion [21]. The circulating HS is presumably shed from the endothelial GCX among other sources, suggesting a mechanistic linkage between inflammatory-mediated ESL perturbation and organ injury.

HS is selectively cleaved from its associated proteoglycan into varying fragment lengths by heparanase-1 (HPSE), the only known mammalian endoglucuronidase to have enzymatic activity on HS [22]. Consistent with the observed elevation of plasma HS levels described above during systemic inflammation, HPSE levels and enzymatic activity are concomitantly elevated in organs injured during inflammation [4,23]. Secreted by activated leukocytes, platelets, and endothelial cells $[24,25]$ in the setting of hyperglycemia [26], angiopoietin-2 [27], TNF $\alpha$ [28], or IL-1 $\beta$ [28], HPSE acts at the GCX to cleave HS from its associated proteoglycan into various fragment lengths $[29,30]$ (Figure 1). This HS trimming releases stereotactically associated proteins (e.g., chemokines, growth factors) into circulation and the local microenvironment [27]. Now free, these proteins activate a myriad of receptors that propagate inflammation, endothelial permeability, and leukocyte transcytosis [31]. Soluble HS fragments can also independently 
serve as damage-associated molecular patterns recognized by tolllike receptor 4 (TLR4), resulting in the unpropitious activation of NF-kB, increased expression of cytokines and cell adhesion molecules, and mitochondrial dysfunction [32-34]. Conversely, Zhang Y et al. [35] demonstrated that soluble HS of varying lengths attenuate circulating histone-mediated damage of human pulmonary microvascular endothelial cells in vitro independently of TLR-signaling. Thus, the local effects of soluble HS are likely contextually dependent on the characteristics of the HS fragments present, the presence of bound signaling molecules, and the surrounding inflammatory milieu.

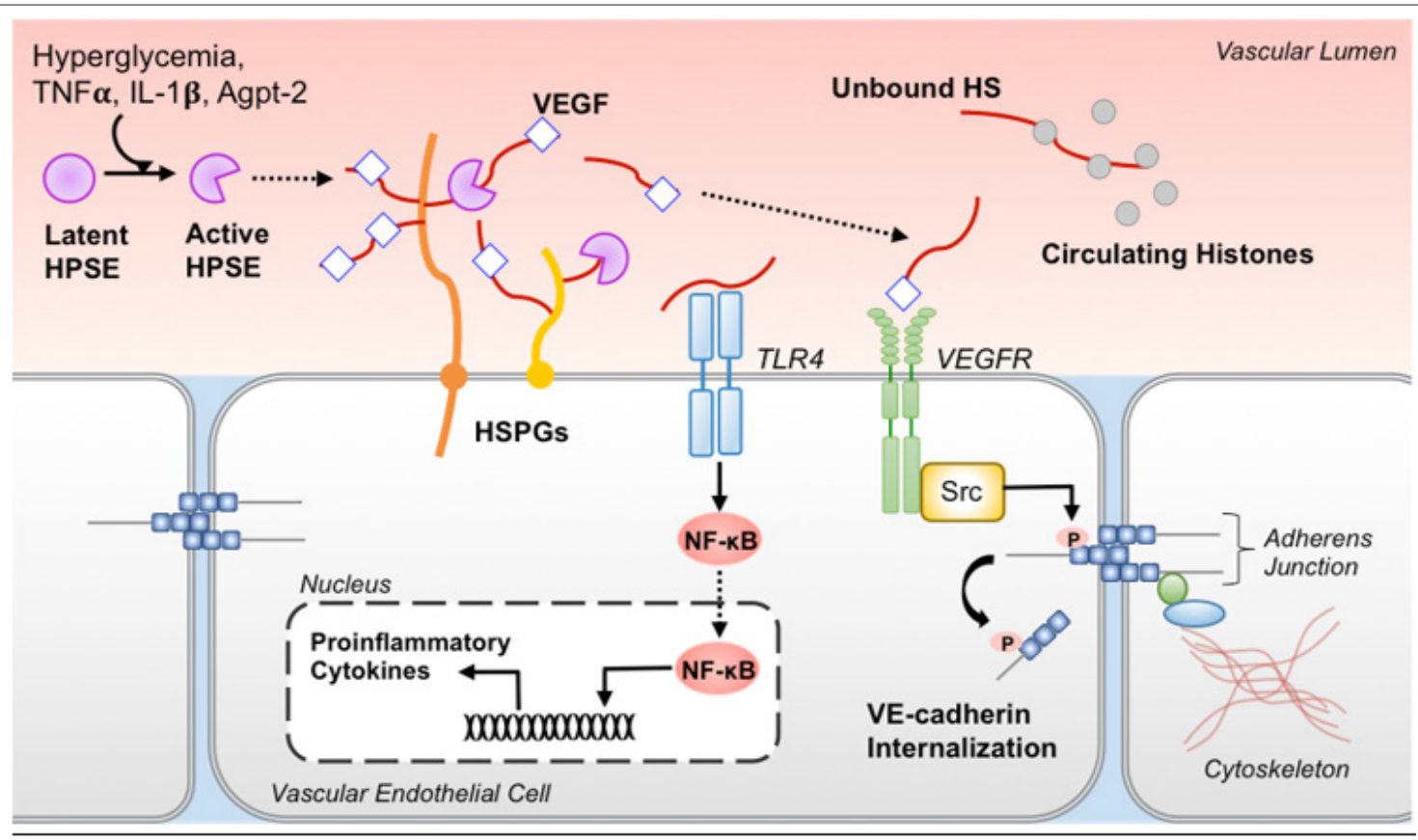

Figure 1: Role of soluble HS during inflammation. Enzymatically active HPSE cleaves HS bound to HSPGs, which releases HS fragments and stereotactically associated chemokines and growth factors (e.g., VEGF) into circulation and the local microenvironment. Soluble HS fragments are then capable of initiating receptor-mediated signaling by facilitating the interaction of the bound growth factor or cytokine with its respective receptor. As an example, HS facilitates VEGF-binding to the VEGF receptor leading to SRC-dependent phosphorylation of VE-cadherin. This increases VEcadherin internalization resulting in destabilization of intercellular junctions. Unbound HS fragments also serve as damage-associated molecular patterns recognized by TLR4, which results in NF-kB-mediated proinflammatory cytokine production. By contrast, unbound HS binds circulating histones, making them less available to propagate inflammation.

\section{Role of Hyaluronan}

Adults admitted to the intensive care unit with septic shock demonstrate elevated plasma HA levels compared to controls [19]. Schmidt et al. [18] similarly reported elevated circulating HA fragments in patients with lung injury. This group went on to describe that in two adult cohorts admitted for sepsis or acute respiratory distress syndrome, increased urinary levels of HA early in the presentation of systemic illness were predictive of the development of acute kidney injury and in-hospital mortality [36]. Taken together, these data suggest that soluble HA and its degradation products promote proinflammatory signaling that culminates in organ dysfunction.

HA is shed from the GCX and further degraded by hyaluronidase and/or reactive oxygen species released from activated leukocytes, resulting in the formation of low molecular weight (LMW)-HA [37]. LMW-HA fragments produce a proinflammatory cellular phenotype, demonstrated by the activation of the M1 phenotype in macrophages in vitro [38,39] and exacerbation of lung injury in in vivo models of airway hyperreactivity [40]. HA signaling appears to be fragment size-dependent as soluble HMW-HA promotes an anti-inflammatory M2 phenotype in macrophages [38]. Several endothelial cell surface receptors that recognize LMWHA have been identified, including TLR4, CD44, and the receptor for HA-mediated motility (RHAMM) (Figure 2). Human dermal microvascular endothelial cells treated with LMW-HA upregulate proinflammatory cytokine production in a TLR4-dependent manner [41]. Evidence for this signaling pathway is supported by the protection of TLR4 knockout mice from LMW-HA-induced lung injury [42]. The interaction of HA with cell surface CD44 has been extensively studied, demonstrating a wide array of signaling cascades terminating in cytokine upregulation, cytoskeletal remodeling, and cell proliferation that can occur independently of or in concert with RHAMM [43]. The role of RHAMM in HA signaling appears to be more nuanced as its expression depends on various factors, including induction by CD44/protein kinase $\mathrm{C} \delta$ activation [44]. After being expressed on the cell membrane, RHAMM binds with soluble LMW-HA, thereby activating the ERK1/2-MAP kinase pathway to promote cellular proliferation [45] and Ras/Raf/Rac to effect cytoskeletal rearrangement [43]. 


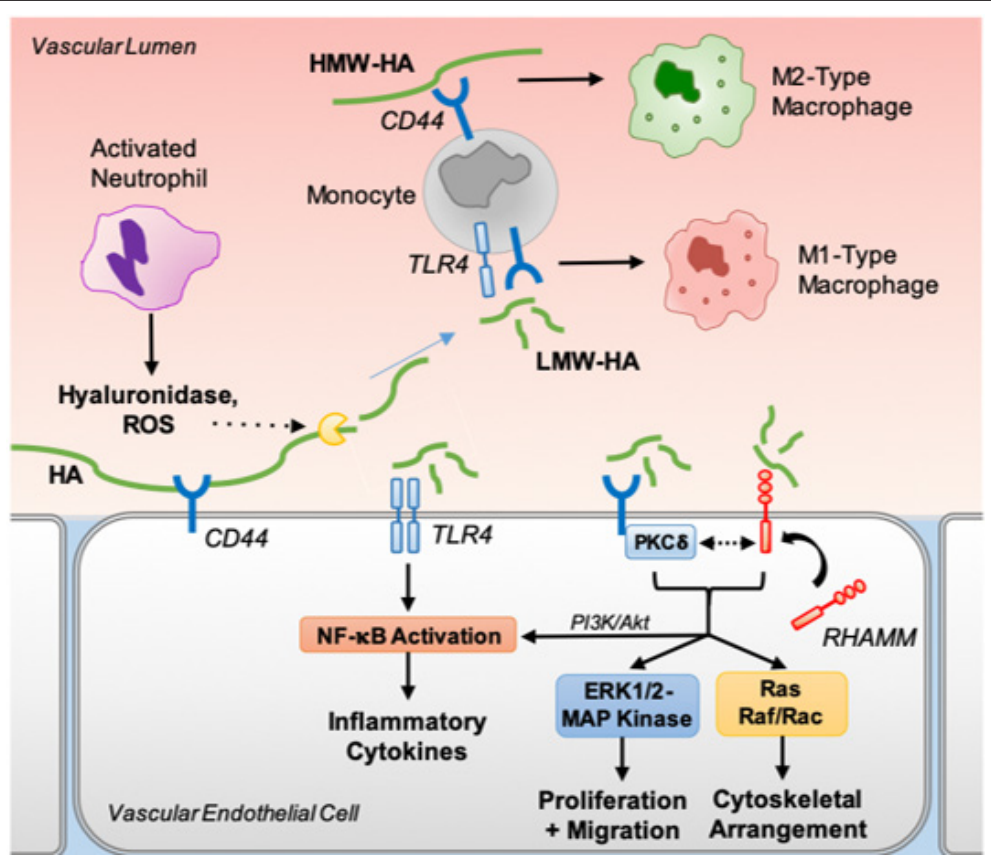

Figure 2: Role of soluble HA in inflammation. ESL-bound HA is degraded in inflammatory states by hyaluronidase or ROS released by activated neutrophils. Soluble LMW-HA binds via CD44 and/or TLR4 to produce a proinflammatory M1-type macrophage, whereas HMW-HA binds via CD44 to promote an anti-inflammatory M2 macrophage phenotype. LMW-HA also activates TRL4 signaling in endothelial cells that results in NF$\mathbf{k B}$ activation and proinflammatory cytokine production. Additionally, LMW-HA can bind to and initiate CD44 signaling, which, via PKC $\delta$, recruits RHAMM to the cell surface. CD44 signaling, both independently and in concert with RHAMM, terminates in cytokine upregulation (via PI3K/Akt/ NF-kB), cell proliferation and migration (via ERK1/2-MAP kinase) and cytoskeletal rearrangement (via Ras/Raf/Rac).

\section{Role of Other Glycosaminoglycans}

The other GAGs expressed in the endothelial GCX have received much less attention, limiting our understanding of their role in the inflammatory response. When shed, chondroitin sulfate appears to exhibit anti-inflammatory properties through histone-binding and NF-kB downregulation $[46,47]$. However, soluble chondroitin sulfates may also exhibit untoward antibacterial peptide inhibition in the setting of sepsis [48]. Dermatan sulfate may exhibit more tissue-specific local inflammatory regulation by mediating FGFRdependent cell proliferation and NF-kB activation with resultant increased ICAM-1 expression in and around epidermal wounds $[49,50]$. Keratan sulfate does not have a known role in inflammatory signaling at present.

\section{Role of Surface Heparan Sulfate Proteoglycans}

The HSPGs are the only known endothelial surface-anchored proteoglycans, making them integral in maintaining GCX integrity and endothelial responsiveness to luminal physical and biochemical stimuli. Loss of endothelial surface expression of HSPGs and their covalently bound GAGs therefore has dire consequences on shear stress mechanosignaling and other cellular signaling mechanisms that regulate vasoactivity, vascular permeability, and endothelial cell homeostasis. Clinical studies bear this to be true. Circulating levels of Syn, particularly Syn1, have repeatedly been shown to significantly rise in systemic inflammatory conditions like trauma $[20,51,52]$ sepsis $[48,53]$ and burns $[54,55]$ and correlate with illness severity $[48,51,54,56]$. Though the glypicans have been less well studied, circulating glypican levels are elevated in the setting of sepsis and correlate with disease severity [57]. Adults with severe direct lung injury from pneumonia also demonstrate elevated levels of plasma glypican [58].

Mechanosensing of vascular shear stress to the endothelial cytoskeleton is primarily mediated through the HSPGs [59] (Figure 3). Syn1 and Syn4, in particular, directly influence cytoskeletal remodeling through syntenin and synectin linkages to the actin cytoskeleton [60-64]. As blood flow shear stress deforms the GCX, Syn transmits this torque to the actin cytoskeleton [65] via, in part, activation of RhoA GTPases [66]. Due to the intricate cytoplasmic connections of the cytoskeleton to adherens and gap junctions and intracellular organelles, the surface Syn-mechanotransduction becomes "decentralized" and propagates throughout the cytoplasm [67]. The resultant cytoskeletal reorganization influences cellular shape and distribution along the vasculature, inter- and intracellular molecular transport, cell-cell adhesions, and nuclear activation [65,67]. Syn 4 also activates protein kinase $\mathrm{C} \alpha$ in a calcium-independent fashion to maintain proper cytoskeletal stress fiber alignment in response to blood flow $[60,68]$. Moreover, Syn4, through its interaction with Rho, activates focal adhesion kinase to maintain focal adhesion integrity [69]. Thus, loss of Syn expression from the ESL would be expected to culminate in destabilized cytoskeletal arrangement with poor endothelial responsiveness to changes in surface shear as demonstrated by Baeyens et al. [70] Degradation of the HSPGs appears to also significantly alter the endothelium's ability to regulate vasoreactivity to vascular shear stress by disrupting eNOS activation. Voyvodic et al. [66] have 
shown that loss of Syn 1 from the GCX suppresses the endothelial cell's ability to activate the PI3/Akt pathway in response to luminal shear, resulting in decreased phosphorylated Akt. Dimmeler et al. [71] have demonstrated that eNOS is activated in response to Akt phosphorylation. Taken together, the results of these studies suggest that loss of Syn 1 expression from the GCX may result in decoupling of eNOS activation from vascular blood flow. HS trimming by heparinase III, a bacterial enzyme with nonspecific HS-degrading activity at the ESL, has also been shown to abrogate shear-induced eNOS activation [59,72]. Likewise, glypican influences caveolae protein 1 expression that subsequently regulates eNOS expression in caveolae [73-75]. Thus, deranged glypican expression suppresses eNOS activity in response to shear via decreased eNOS expression $[9,76]$. Interestingly, $\mathrm{HA}$ also participates in mechanosignaling to influence eNOS activation [77,78]. Thus, removal of HSPGs and HA impairs shear-induced vasoactivity of the endothelial cell by attenuating eNOS expression and activation.

HSPGs protect the endothelium from leukocytes and platelets by shielding cell adhesion molecules and glycoproteins used by these circulating cells for endothelial attachment [79,80]. HPSEmediated removal of HS from Syn opens the proteoglycan up to the enzymatic activity of MMPs [81-83], thereby exposing P- and E-selectins and VCAM-1, PECAM-1, and ICAM-1. Now available, circulating leukocytes and platelets adhere to these cell adhesion molecules and become activated $[4,80,84,85]$. Indeed, the result of loss of Syn 1 endothelial surface expression has been shown to promote a proinflammatory phenotype that is abrogated by reexpression of Syn1 [66]. Similarly, treatment of various endothelial cell lines with heparinase III has been shown to significantly increase leukocyte adhesion with resultant tissue injury $[4,86]$. Release of chemokines from the cell surface during GCX degradation into the local microenvironment may serve to attract leukocytes to and through the endothelium. Conversely, chemokine release from the ESL may also act as a mechanism to reduce leukocyte attachment to the endothelium [87]. Furthermore, like HSPGs, cell adhesion molecules may undergo enzymatic cleavage by MMPs [88]. Shapiro et al. [89] found plasma levels of the soluble form of several cell adhesion molecules (e.g., sE-selectin, sICAM-1, and sVCAM-1) to be significantly elevated in adults presenting to the emergency department with sepsis. While the purpose for cell adhesion molecule cleavage is not entirely clear, it may provide a protective mechanism for the endothelium to limit leukocyte attachment and activation or it may serve as an essential step in the active invasion of the leukocyte through the endothelium by dissociating from the ESL. Thus, the degree to which the GCX prevents or aides in leukocyte chemotaxis, attachment, and activation during inflammatory-related ESL disruption may be dictated by the degree of remaining HS and cell adhesion molecule expression after GCX degradation [90].

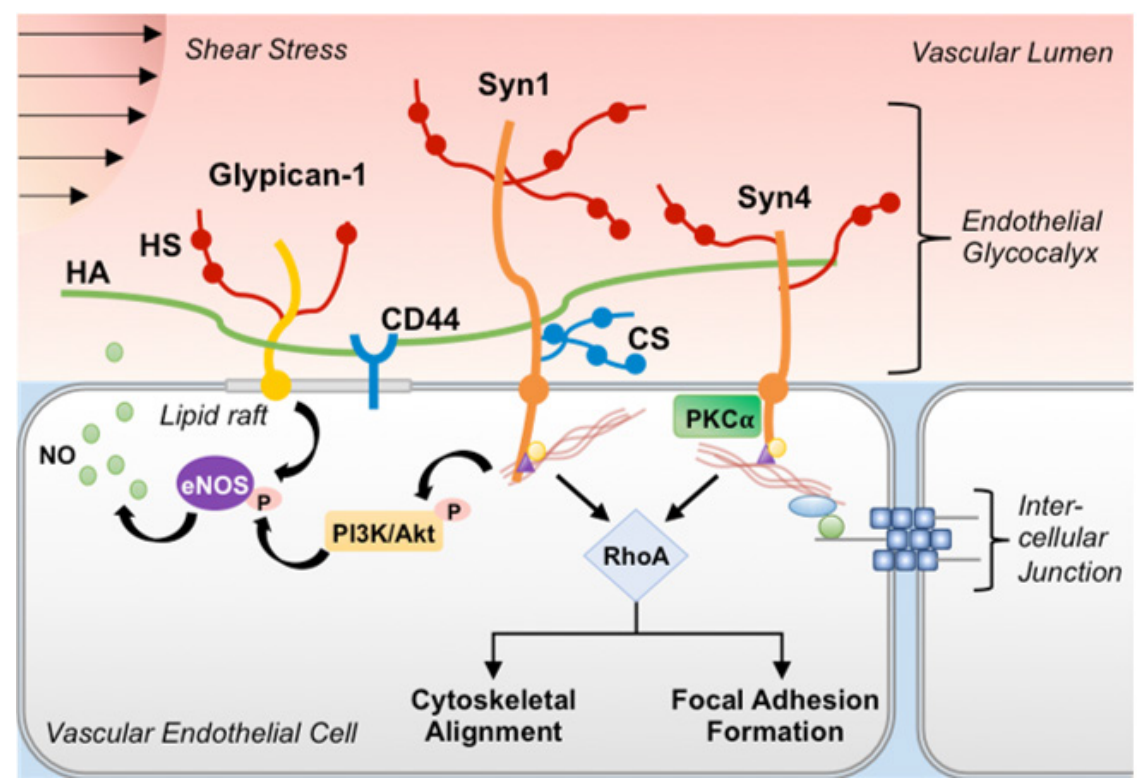

Figure 3: GCX-mediated mechanosignaling. The cytoplasmic domain of Syn is associated with the actin cytoskeletal network via linkages with syntenin and synectin, which allows for propagation of mechanotransduction signaling from the cellular surface to intercellular junctions and throughout the cytoplasm. In response to shear stress, Syn initiates cytoskeletal alignment and focal adhesion formation via activation of RhoA. In some cell types, Syn1 may also regulate shear stress-induced activation of PI3K/Akt signaling. Glypican, HS, and HA mediate cellular activation of eNOS and NO production in response to shear stress via associations with caveolae/lipid raft regions that are enriched with signaling molecules (e.g., inactive eNOS). Loss of any or all of these GCX constituents impairs the endothelial cell response to intra-luminal forces.

Lastly, decreased HSPG expression can disrupt critical receptormediated signaling pathways important for maintaining endothelial homeostasis. Downregulation of Syn1 in murine glomerular endothelial cells affects VEGF-VEGFR2 signaling with resultant reduction in downstream Akt, ERK1/2, and Rac1 activation [91]. FGF2-FGFR signaling is heavily influenced by Syn 4 stabilization of the growth factor-receptor complex to affect a multitude of complex intracellular pathways that result in maintenance of HS expression 
in the GCX and stabilization of endothelial cell-cell adhesions [92]. Constitutive expression of Syn 4 also contributes to the tightly controlled release of angiopoietin-2 (Agpt-2) from the endothelium [93]. Agpt-2 is a cytokine that antagonizes the endothelial cell surface Tie2 receptor, resulting in destabilization of cell-cell junctions [94]. Thus, loss of Syn-4 ESL expression may contribute to dysregulated FGFR signaling and Agpt-2 release that would result in increased endothelial permeability and impaired GCX restoration. The role that glypican disruption plays in endothelial cell receptor-mediated signaling during inflammation remains to be studied [95].

\section{Therapeutic Strategies Targeting GCX Restoration}

As our knowledge of the signaling mechanisms influenced by the GCX is continually growing, so too is the development of novel strategies to (1) prevent GCX damage, (2) inhibit the downstream detrimental signaling cascades initiated by GCX shedding, and/or (3) promote GCX restoration. Over the past two decades, various studies have demonstrated the impact that fluid resuscitation strategies have on clinical outcomes, due in part to the recognition of the role of endothelial GCX integrity in mediating organ function. Well-intentioned goal-directed clinical practices (e.g., early highvolume crystalloid resuscitation for hemorrhagic or septic shock) may actually worsen clinical outcomes, [96,97] in part through GCX perturbation $[98,99]$. The use of fresh frozen plasma (FFP) as a resuscitation fluid has received much attention due to its ability to upregulate GCX synthesis, promote repair of EC junctions, and restore microvascular function after hemorrhagic shock [98,100102]. More recently, the GCX-protective benefits of FFP resuscitation have been attributed to its adiponectin [103], ATIII [104-106]. Other therapies that demonstrate GCX-protection include hydrocortisone [104] via mitigation of TNF $\alpha$-induced endothelial activation; dexamethasone [107] and sphingosine-1-phosphate[8] by downregulating endothelial cell MMP expression; doxycycline [108] via MMP inhibition; and heparin/heparan sulfate mimetics derivatives $[4,109,110]$ via HPSE inhibition. To prevent aberrant downstream signaling events after GCX injury, purified HMW-HA may be administered systemically [111]. Lastly, ESL regeneration may be promoted by FFP as described above; sphingosine-1phosphate by increasing ESL HS expression [112]; sulodexide $[113,114]$ by re-coating the ESL with highly purified GAGs; and FGF-receptor agonists by promoting exostosin-1 expression and HS synthesis [115]. Although promising, these therapies remain theoretical at present and require further study with prospective randomized clinical trials.

\section{Conclusions}

Since the presence of a protein-rich layer on the vascular endothelium was first proposed in 1940 [116], our understanding of the pivotal role of the endothelial GCX in maintaining vascular integrity has grown immensely. It is clear that disruption of the endothelial GCX by inflammatory conditions initiates a cascade of signaling events that culminate in inflammation propagation and vascular hyperpermeability. Each major component of the GCX structure, namely HS, HA, and the HSPGs, contributes uniquely to the function of the ESL both in health and inflammatory pathophysiology. However, further study is needed to more clearly understand how GCX-component degradation contributes to endotheliopathy in the setting of inflammation. There is compelling evidence to suggest that strategies targeting the prevention of GCX damage and the restoration of its integrity will improve vascular function, which has the potential to meaningfully improve clinical outcomes. Given the progress in the field of end othelial GCX research over the last several years, we anticipate significant translational developments of therapies aimed at protecting the GCX in the years to come [116].

\section{References}

1. Reitsma S, Slaaf DW, Vink H, van Zandvoort MA, oude Egbrink MG (2007) The endothelial glycocalyx: composition, functions, and visualization. Pflugers Arch 454(3): 345-359.

2. Ushiyama A, Kataoka H, Iijima T (2016) Glycocalyx and its involvement in clinical pathophysiologies. J Intensive Care 4(1): 59.

3. Ledin J, Staatz W, Li JP, Gotte M, Selleck S, et al. (2004) Heparan sulfate structure in mice with genetically modified heparan sulfate production. J Biol Chem 279(41): 42732-42741.

4. Schmidt EP, YangY, Janssen WJ, Gandjeva A, Perez MJ, et al. (2012) The pulmonary endothelial glycocalyx regulates neutrophil adhesion and lung injury during experimental sepsis. Nat Med 18(8): 1217-1223.

5. Kim CW, Goldberger OA, Gallo RL, Bernfield M (1994) Members of the syndecan family of heparan sulfate proteoglycans are expressed in distinct cell-, tissue-, and development-specific patterns. Mol Biol Cell 5(7): 797-805.

6. Tkachenko E, Rhodes JM, Simons M (2005) Syndecans: new kids on the signaling block. Circ Res 96(5): 488-500.

7. Farrugia BL, Lord MS, Melrose J, Whitelock JM (2018) The Role of Heparan Sulfate in Inflammation, and the Development of Biomimetics as Anti-Inflammatory Strategies. J Histochem Cytochem 66(4): 321-336.

8. Zeng Y, Tarbell JM (2014) The adaptive remodeling of endothelial glycocalyx in response to fluid shear stress. PLoS One 9(1): e86249.

9. Zeng Y, Liu J (2016) Role of glypican-1 in endothelial NOS activation under various steady shear stress magnitudes. Exp Cell Res 348(2): 184189.

10. Kinsella MG, Bressler SL, Wight TN (2004) The regulated synthesis of versican, decorin, and biglycan: extracellular matrix proteoglycans that influence cellular phenotype. Crit Rev Eukaryot Gene Expr 14(3): 203234.

11. Kolarova H, Ambruzova B, Svihalkova Sindlerova L, Klinke A, Kubala L (2014) Modulation of endothelial glycocalyx structure under inflammatory conditions. Mediators Inflamm 2014: 694312.

12. Lindahl U, Kusche Gullberg M, Kjellen L (1998). Regulated diversity of heparan sulfate. J Biol Chem 273(39): 24979-24982.

13. Woodcock TE, Woodcock TM (2012) Revised Starling equation and the glycocalyx model of transvascular fluid exchange: an improved paradigm for prescribing intravenous fluid therapy. Br J Anaesth 108(3): 384-394.

14. Afratis NA, Nikitovic D, Multhaupt HA, Theocharis AD, Couchman JR, et al. (2017) Syndecans - key regulators of cell signaling and biological functions. FEBS J 284(1): 27-41. 
15. Giuffre L, Cordey AS, Monai N, Tardy Y, Schapira M, et al. (1997) Monocyte adhesion to activated aortic endothelium: role of L-selectin and heparan sulfate proteoglycans. J Cell Biol 136(4): 945-956.

16. Gotte M (2003) Syndecans in inflammation. FASEB J 17(6): 575-591.

17. Yen W, Cai B, Yang J, Zhang L, Zeng M, et al. (2015) Endothelial surface glycocalyx can regulate flow-induced nitric oxide production in microvessels in vivo. PLoS One 10(1): e0117133.

18. Schmidt EP, Li G, Li L, Fu L, Yang Y, et al. (2014) The circulating glycosaminoglycan signature of respiratory failure in critically ill adults. J Biol Chem 289(12): 8194-8202.

19. Nelson A, Berkestedt I, Bodelsson M (2014). Circulating glycosaminoglycan species in septic shock. Acta Anaesthesiol Scand 58(1): 36-43.

20. Rahbar E, Cardenas JC, Baimukanova G, Usadi B, Bruhn R, et al. (2015) Endothelial glycocalyx shedding and vascular permeability in severely injured trauma patients. J Transl Med 13: 117.

21. Rehm M, Bruegger D, Christ F, Conzen P, Thiel M, et al. (2007) Shedding of the endothelial glycocalyx in patients undergoing major vascular surgery with global and regional ischemia. Circulation 116(17): 1896-1906.

22. Vlodavsky I, Friedmann Y (2001) Molecular properties and involvement of heparanase in cancer metastasis and angiogenesis. J Clin Invest 108(3): 341-347

23. Lygizos MI, Yang Y, Altmann CJ, Okamura K, Hernando AA, et al. (2013) Heparanase mediates renal dysfunction during early sepsis in mice. Physiol Rep 1(6): e00153.

24. Godder K, Vlodavsky I, Eldor A, Weksler BB, Haimovitz Freidman A, et al. (1991) Heparanase activity in cultured endothelial cells. J Cell Physiol 148(2): 274-280.

25. Vlodavsky I, Eldor A, Haimovitz Friedman A, Matzner Y, Ishai Michaeli R, et al. (1992) Expression of heparanase by platelets and circulating cells of the immune system: possible involvement in diapedesis and extravasation. Invasion Metastasis 12(2): 112-127.

26. Wang F, Wang Y, Kim MS, Puthanveetil P, Ghosh S, et al. (2010) Glucose-induced endothelial heparanase secretion requires cortical and stress actin reorganization. Cardiovasc Res 87(1): 127-136.

27. Lukasz A, Hillgruber C, Oberleithner H, Kusche Vihrog K, Pavenstadt $H$, et al. (2017) Endothelial glycocalyx breakdown is mediated by angiopoietin 2. Cardiovasc Res 113(6): 671-680.

28. Chen G, Wang D, Vikramadithyan R, Yagyu H, Saxena U, et al. (2004) Inflammatory cytokines and fatty acids regulate endothelial cell heparanase expression. Biochemistry 43(17): 4971-4977.

29. Vreys V, David G (2007) Mammalian heparanase: what is the message? J Cell Mol Med 11(3): 427-452.

30. Oshima K, Haeger SM, Hippensteel JA, Herson PS, Schmidt EP (2018) More than a biomarker: the systemic consequences of heparan sulfate fragments released during endothelial surface layer degradation (2017 Grover Conference Series). Pulm Circ 8(1): 2045893217745786.

31. Parish CR (2006) The role of heparan sulphate in inflammation. Nat Rev Immunol 6(9): 633-643.

32. Johnson GB, Brunn GJ, Kodaira Y, Platt JL (2002) Receptor-mediated monitoring of tissue well-being via detection of soluble heparan sulfate by Toll-like receptor 4. J Immunol 168(10): 5233-5239.

33. Goodall KJ, Poon IK, Phipps S, Hulett MD (2014) Soluble heparan sulfate fragments generated by heparanase trigger the release of pro-inflammatory cytokines through TLR-4. PLoS One 9(10): e109596.

34. Martin L, Peters C, Schmitz S, Moellmann J, Martincuks A, et al. (2015) Soluble Heparan Sulfate in Serum of Septic Shock Patients Induces Mitochondrial Dysfunction in Murine Cardiomyocytes. Shock 44(6): 569577.

35. Zhang Y, Haeger SM, Yang Y, Dailey KL, Ford JA, et al. (2017) Circulating Heparan Sulfate Fragments Attenuate Histone-Induced Lung Injury In- dependently of Histone Binding. Shock 48(6): 666-673.

36. Schmidt EP, Overdier KH, Sun X, Lin L, Liu X, et al. (2016) Urinary Glycosaminoglycans Predict Outcomes in Septic Shock and Acute Respiratory Distress Syndrome. Am J Respir Crit Care Med 194(4): 439-449.

37. Litwiniuk M, Krejner A, Speyrer MS, Gauto AR, Grzela T (2016) Hyaluronic Acid in Inflammation and Tissue Regeneration. Wounds 28(3): 78-88.

38. Rayahin JE, Buhrman JS, Zhang Y, Koh TJ, Gemeinhart RA (2015) High and low molecular weight hyaluronic acid differentially influence macrophage activation. ACS Biomater Sci Eng 1(7): 481-493.

39. McKee CM, Penno MB, Cowman M, Burdick MD, Strieter RM, et al. (1996) Hyaluronan (HA) fragments induce chemokine gene expression in alveolar macrophages. The role of HA size and CD44. J Clin Invest 98(10): 2403-2413.

40. Garantziotis S, Li Z, Potts EN, Kimata K, Zhuo L, et al. (2009) Hyaluronan mediates ozone-induced airway hyperresponsiveness in mice. J Biol Chem 284(17): 11309-11317.

41. Taylor KR, Trowbridge JM, Rudisill JA, Termeer CC, Simon JC, et al. (2004) Hyaluronan fragments stimulate endothelial recognition of injury through TLR4. J Biol Chem 279(17): 17079-17084.

42. Garantziotis S, Li Z, Potts EN, Lindsey JY, Stober VP, et al. (2010) TLR4 is necessary for hyaluronan-mediated airway hyperresponsiveness after ozone inhalation. Am J Respir Crit Care Med 181(7): 666-675.

43. Jordan AR, Racine RR, Hennig MJ, Lokeshwar VB (2015. The Role of CD44 in Disease Pathophysiology and Targeted Treatment. Front Immunol 6: 182.

44. Park D, Kim Y, Kim H, Kim K, Lee YS, et al. (2012) Hyaluronic acid promotes angiogenesis by inducing RHAMM-TGFbeta receptor interaction via CD44-PKCdelta. Mol Cells 33(6): 563-574.

45. Misra S, Hascall VC, Markwald RR, Ghatak S (2015) Interactions between Hyaluronan and Its Receptors (CD44, RHAMM) Regulate the Activities of Inflammation and Cancer. Front Immunol 6: 201.

46. Nagano F, Mizuno T, Mizumoto S, Yoshioka K, Takahashi K, et al. (2018) Chondroitin sulfate protects vascular endothelial cells from toxicities of extracellular histones. Eur J Pharmacol 826: 48-55.

47. Herrero Beaumont G, Marcos ME, Sanchez Pernaute O, Granados R, Ortega L, et al. (2008) Effect of chondroitin sulphate in a rabbit model of atherosclerosis aggravated by chronic arthritis. Br J Pharmacol 154(4): 843-851.

48. Nelson A, Berkestedt I, Schmidtchen A, Ljunggren L, Bodelsson M (2008) Increased levels of glycosaminoglycans during septic shock: relation to mortality and the antibacterial actions of plasma. Shock 30(6): 623-627.

49. Penc SF, Pomahac B, Winkler T, Dorschner RA, Eriksson E, et al. (1998) Dermatan sulfate released after injury is a potent promoter of fibroblast growth factor-2 function. J Biol Chem 273(43): 28116-28121.

50. Penc SF, Pomahac B, Eriksson E, Detmar M, Gallo RL (1999) Dermatan sulfate activates nuclear factor-kappab and induces endothelial and circulating intercellular adhesion molecule-1. J Clin Invest 103(9): 13291335.

51. Gonzalez Rodriguez E, Ostrowski SR, Cardenas JC, Baer LA, Tomasek JS, et al. (2017) Syndecan-1: A Quantitative Marker for the Endotheliopathy of Trauma. J Am Coll Surg 225(3): 419-427.

52. Horowitz A, Tkachenko E, Simons M (2002) Fibroblast growth factor-specific modulation of cellular response by syndecan-4. J Cell Biol 157(4): 715-725.

53. Anand D, Ray S, Srivastava LM, Bhargava S (2016) Evolution of serum hyaluronan and syndecan levels in prognosis of sepsis patients. Clin Biochem 49(10-11): 768-776.

54. Osuka A, Kusuki H, Yoneda K, Matsuura H, Matsumoto H, et al. (2018) Glycocalyx Shedding is Enhanced by Age and Correlates with Increased Fluid Requirement in Patients with Major Burns. Shock 50(1): 60-65. 
55. Luker JN, Vigiola Cruz M, Carney BC, Day A, Moffatt LT, et al. (2018) Shedding of the endothelial glycocalyx is quantitatively proportional to burn injury severity. Ann Burns Fire Disasters 31(1): 17-22.

56. Ostrowski SR, Berg RM, Windelov NA, Meyer MA, Plovsing RR, et al. (2013) Coagulopathy, catecholamines, and biomarkers of endothelial damage in experimental human endotoxemia and in patients with severe sepsis: a prospective study. J Crit Care 28(5): 586-596.

57. Fisher J, Linder A, Bentzer P (2019) Elevated plasma glypicans are associated with organ failure in patients with infection. Intensive Care Med $\operatorname{Exp} 7(1): 2$.

58. Chen C, Shi L, Li Y, Wang X, Yang S (2016) Disease-specific dynamic biomarkers selected by integrating inflammatory mediators with clinical informatics in ARDS patients with severe pneumonia. Cell Biol Toxicol 32(3): 169-184.

59. Florian JA, Kosky JR, Ainslie K, Pang Z, Dull RO, et al. (2003) Heparan sulfate proteoglycan is a mechanosensor on endothelial cells. Circ Res 93(10): e136-142.

60. Simons M, Horowitz A (2001) Syndecan-4-mediated signalling. Cell Signal 13(12): 855-862.

61. Elfenbein A, Simons M (2013) Syndecan-4 signaling at a glance. J Cell Sci 126(Pt 17): 3799-3804.

62. Sulka B, Lortat Jacob H, Terreux R, Letourneur F, Rousselle P (2009) Tyrosine dephosphorylation of the syndecan-1 PDZ binding domain regulates syntenin-1 recruitment. J Biol Chem 284(16): 10659-10671.

63. Grootjans JJ, Zimmermann P, Reekmans G, Smets A, Degeest G, et al. (1997) Syntenin, a PDZ protein that binds syndecan cytoplasmic domains. Proc Natl Acad Sci U S A 94(25): 13683-13688.

64. Bass MD, Humphries MJ (2002) Cytoplasmic interactions of syndecan-4 orchestrate adhesion receptor and growth factor receptor signalling. Biochem J 368(Pt 1): 1-15.

65. Thi MM, Tarbell JM, Weinbaum S, Spray DC (2004) The role of the glycocalyx in reorganization of the actin cytoskeleton under fluid shear stress: a "bumper-car" model. Proc Natl Acad Sci USA 101(47): 16483-16488.

66. Voyvodic PL, Min D, Liu R, Williams E, Chitalia V, et al. (2014) Loss of syndecan-1 induces a pro-inflammatory phenotype in endothelial cells with a dysregulated response to atheroprotective flow. J Biol Chem 289(14): 9547-9559.

67. Helmke BP, Davies PF (2002) The cytoskeleton under external fluid mechanical forces: hemodynamic forces acting on the endothelium. Ann Biomed Eng 30(3): 284-296.

68. Dovas A, Yoneda A, Couchman JR (2006) PKCbeta-dependent activation of RhoA by syndecan-4 during focal adhesion formation. J Cell Sci 119(Pt 13): 2837-2846.

69. Wilcox Adelman SA, Denhez F, Goetinck PF (2002) Syndecan-4 modulates focal adhesion kinase phosphorylation. J Biol Chem 277(36): 32970-32977.

70. Baeyens N, Mulligan Kehoe MJ, Corti F, Simon DD, Ross TD, et al. (2014) Syndecan 4 is required for endothelial alignment in flow and atheroprotective signaling. Proc Natl Acad Sci U S A 111(48): 17308-17313.

71. Dimmeler S, Fleming I, Fisslthaler B, Hermann C, Busse R, et al. (1999) Activation of nitric oxide synthase in endothelial cells by Akt-dependent phosphorylation. Nature 399(6736): 601-605

72. Pahakis MY, Kosky JR, Dull RO, Tarbell JM (2007) The role of endothelial glycocalyx components in mechanotransduction of fluid shear stress. Biochem Biophys Res Commun 355(1): 228-233.

73. Echarri A, Del Pozo MA (2015) Caveolae - mechanosensitive membrane invaginations linked to actin filaments. J Cell Sci 128(15): 2747-2758.

74. Varshney P, Yadav V, Saini N (2016) Lipid rafts in immune signalling: current progress and future perspective. Immunology 149(1): 13-24.
75. Harding IC, Mitra R, Mensah SA, Herman IM, Ebong EE (2018) Pro-atherosclerotic disturbed flow disrupts caveolin-1 expression, localization, and function via glycocalyx degradation. J Transl Med 16(1): 364.

76. Ebong EE, Lopez Quintero SV, Rizzo V, Spray DC, Tarbell JM (2014) Shear-induced endothelial NOS activation and remodeling via heparan sulfate, glypican-1, and syndecan-1. Integr Biol (Camb) 6(3): 338-347.

77. Mochizuki S, Vink H, Hiramatsu O, Kajita T, Shigeto F, et al. (2003) Role of hyaluronic acid glycosaminoglycans in shear-induced endothelium-derived nitric oxide release. Am J Physiol Heart Circ Physiol 285(2): H722726

78. Kumagai R, Lu X, Kassab GS (2009) Role of glycocalyx in flow-induced production of nitric oxide and reactive oxygen species. Free Radic Biol Med 47(5): 600-607.

79. Constantinescu AA, Vink H, Spaan JA (2003) Endothelial cell glycocalyx modulates immobilization of leukocytes at the endothelial surface. Arterioscler Thromb Vasc Biol 23(9): 1541-1547.

80. Vink H, Constantinescu AA, Spaan JA (2000) Oxidized lipoproteins degrade the endothelial surface layer: implications for platelet-endothelial cell adhesion. Circulation 101(13): 1500-1502.

81. Ramani VC, Pruett PS, Thompson CA, DeLucas LD, Sanderson RD (2012) Heparan sulfate chains of syndecan-1 regulate ectodomain shedding. J Biol Chem 287(13): 9952-9961.

82. Yang Y, Macleod V, Miao HQ, Theus A, Zhan F, et al. (2007) Heparanase enhances syndecan-1 shedding: a novel mechanism for stimulation of tumor growth and metastasis. J Biol Chem 282(18): 13326-13333.

83. Purushothaman A, Chen L, Yang Y, Sanderson RD (2008) Heparanase stimulation of protease expression implicates it as a master regulator of the aggressive tumor phenotype in myeloma. J Biol Chem 283(47): 32628-32636

84. Mulivor AW, Lipowsky HH (2009) Inhibition of glycan shedding and leukocyte-endothelial adhesion in postcapillary venules by suppression of matrixmetalloprotease activity with doxycycline. Microcirculation 16(8): 657-666

85. van Griensven M, Probst C, Muller K, Hoevel P, Pape HC (2006) Leukocyte-endothelial interactions via ICAM-1 are detrimental in polymicrobial sepsis. Shock 25(3): 254-259.

86. McDonald KK, Cooper S, Danielzak L, Leask RL (2016) Glycocalyx Degradation Induces a Proinflammatory Phenotype and Increased Leukocyte Adhesion in Cultured Endothelial Cells under Flow. PLoS One 11(12): e0167576.

87. Hayashida K, Parks WC, Park PW (2009) Syndecan-1 shedding facilitates the resolution of neutrophilic inflammation by removing sequestered CXC chemokines. Blood 114(14): 3033-3043.

88. Garton KJ, Gough PJ, Raines EW (2006) Emerging roles for ectodomain shedding in the regulation of inflammatory responses. J Leukoc Biol 79(6): 1105-1116.

89. Shapiro NI, Schuetz P, Yano K, Sorasaki M, Parikh SM, et al. (2010) The association of endothelial cell signaling, severity of illness, and organ dysfunction in sepsis. Crit Care 14(5): R182.

90. Wang L, Fuster M, Sriramarao P, Esko JD (2005) Endothelial heparan sulfate deficiency impairs L-selectin- and chemokine-mediated neutrophil trafficking during inflammatory responses. Nat Immunol 6(9): 902-910.

91. Jing Z, Wei Jie Y, Yi Feng ZG, Jing H (2016) Downregulation of Syndecan-1 induce glomerular endothelial cell dysfunction through modulating internalization of VEGFR-2. Cell Signal 28(8): 826-837.

92. Yang X, Liaw L, Prudovsky I, Brooks PC, Vary C, et al. (2015) Fibroblast growth factor signaling in the vasculature. Curr Atheroscler Rep 17(6): 509.

93. Ju R, Zhuang ZW, Zhang J, Lanahan AA, Kyriakides T, et al. (2014) Angiopoietin-2 secretion by endothelial cell exosomes: regulation by the phosphatidylinositol 3-kinase (PI3K)/Akt/endothelial nitric oxide syn- 
thase (eNOS) and syndecan-4/syntenin pathways. J Biol Chem 289(1): 510-519.

94. Leligdowicz A, Richard Greenblatt M, Wright J, Crowley VM, Kain KC (2018) Endothelial Activation: The Ang/Tie Axis in Sepsis. Front Immunol 9: 838.

95. Filmus J, Capurro M, Rast J (2008) Glypicans. Genome Biol 9(5): 224.

96. Self WH, Semler MW, Bellomo R, Brown SM, deBoisblanc BP, et al. (2018) Liberal Versus Restrictive Intravenous Fluid Therapy for Early Septic Shock: Rationale for a Randomized Trial. Ann Emerg Med 72(4): 457 466.

97. Bickell WH, Wall MJ Jr, Pepe PE, Martin RR, Ginger VF, et al. (1994) Immediate versus delayed fluid resuscitation for hypotensive patients with penetrating torso injuries. N Engl J Med 331(17): 1105-1109.

98. Torres Filho IP, Torres LN, Salgado C, Dubick MA (2016) Plasma syndecan-1 and heparan sulfate correlate with microvascular glycocalyx degradation in hemorrhaged rats after different resuscitation fluids. Am J Physiol Heart Circ Physiol 310(11): H1468-1478.

99. Milford EM, Reade MC (2019) Resuscitation fluid choice to preserve the endothelial glycocalyx. Crit Care 23(77): 1-11.

100. Haywood Watson RJ, Holcomb JB, Gonzalez EA, Peng Z, Pati S, et al. (2011) Modulation of syndecan-1 shedding after hemorrhagic shock and resuscitation. PLoS One 6(8): e23530.

101. Kozar RA, Peng Z, Zhang R, Holcomb JB, Pati S, et al. (2011) Plasma restoration of endothelial glycocalyx in a rodent model of hemorrhagic shock. Anesth Analg 112(6): 1289-1295.

102. Vigiola Cruz M, Carney BC, Luker JN, Monger KW, Vazquez JS, et al. (2019) Plasma Ameliorates Endothelial Dysfunction in Burn Injury. J Surg Res 233: 459-466.

103. Deng X, Cao Y, Huby MP, Duan C, Baer L, et al. (2016) Adiponectin in Fresh Frozen Plasma Contributes to Restoration of Vascular Barrier Function After Hemorrhagic Shock. Shock 45(1): 50-54.

104. Chappell D, Hofman Kiefer K, Jacob M, Rehm M, Briegel J, et al. (2009) TNF-alpha induced shedding of the endothelial glycocalyx is prevented by hydrocortisone and antithrombin. Basic Res Cardiol 104(1): 78-89.

105. Chappell D, Dorfler N, Jacob M, Rehm M, Welsch, U, et al. (2010) Glycocalyx protection reduces leukocyte adhesion after ischemia/reperfusion. Shock 34(2): 133-139.
106. Wu, F. and Kozar, R.A. (2019). Fibrinogen Protects Against Barrier Dysfunction Through Maintaining Cell Surface Syndecan-1 In Vitro. Shock 51(6): 740-744.

107. Cui N, Wang H, Long Y, Su L, Liu D (2015) Dexamethasone Suppressed LPS-Induced Matrix Metalloproteinase and Its Effect on Endothelial Glycocalyx Shedding. Mediators Inflamm 2015: 912726.

108. Lipowsky HH, Lescanic A (2013) The effect of doxycycline on shedding of the glycocalyx due to reactive oxygen species. Microvasc Res 90: 80-85.

109. Yini S, Heng Z, Xin A, Xiaochun M (2015) Effect of unfractionated heparin on endothelial glycocalyx in a septic shock model. Acta Anaesthesiol Scand 59(2): 160-169.

110. Abassi Z, Hamoud S, Hassan A, Khamaysi I, Nativ O, et al. (2017) Involvement of heparanase in the pathogenesis of acute kidney injury: nephroprotective effect of PG545. Oncotarget 8(21): 34191-34204.

111. Liu YY, Lee CH, Dedaj R, Zhao H, Mrabat H, et al. (2008) High-molecular-weight hyaluronan--a possible new treatment for sepsis-induced lung injury: a preclinical study in mechanically ventilated rats. Crit Care 12(4): R102.

112. Mensah SA, Cheng MJ, Homayoni H, Plouffe BD, Coury AJ, et al. (2017) Regeneration of glycocalyx by heparan sulfate and sphingosine 1-phosphate restores inter-endothelial communication. PLoS One 12(10): e0186116.

113. Li T, Liu X, Zhao Z, Ni L, Liu C (2017) Sulodexide recovers endothelial function through reconstructing glycocalyx in the balloon-injury rat carotid artery model. Oncotarget 8(53): 91350-91361.

114. Song JW, Zullo JA, Liveris D, Dragovich M, Zhang XF, et al. (2017) Therapeutic Restoration of Endothelial Glycocalyx in Sepsis. J Pharmacol Exp Ther 361(1): 115-121.

115. Yang Y, Haeger SM, Suflita MA, Zhang F, Dailey KL, et al. (2017) Fibroblast Growth Factor Signaling Mediates Pulmonary Endothelial Glycocalyx Reconstitution. Am J Respir Cell Mol Biol 56(6): 727-737.

116. Danielli JF (1940) Capillary permeability and oedema in the perfused frog. J Physiol 98(1): 109-129. 